large amount of heavy ions is present in the corona discharge. The time required for the evolution of the corona pressure is calculated and found to be in agreement with experience. The phenomena of pressure increase in alternating current corona are also explained.

UNIVERSITY OF ILLINOIS,

November 6, I92I.

\title{
The Vibration of Wires in the Corona.
}

\section{By Charles S. Fazel.}

THE vibration of wires carrying a sufficiently high potential to show the corona has been reported by a number of observers. This effect takes place in both the direct and alternating current corona. In view of the close relation between the electric wind, the corona pressure, and this type of vibration, the conditions for its production have been studied. Thus far these studies have been confined to the case of an alternating potential between parallel wires.

It has been found that in order to get maximum vibration the natural frequency of the wire must be the same as that of the impressed potential. However it is necessary that the tension on the wire be small. That is the resonance amplitude is larger with smaller tension.

As the voltage is increased the amplitude increases very abruptly but soon reaches a constant value. This value is maintained as the voltage is increased until a critical voltage is reached where another sharp increase to a constant value is observed. The location of this critical voltage depends on the distance between the wires. The final value of the amplitude is maintained until the arc stage is reached.

The relation between the voltage and the amplitude may be explained on Kunz's theory of the corona pressure. In the first stage the vibration of the wire is due to the momentum of the high mobility carriers. When sufficient electric force is available to get the low mobility, heavy carriers across in the time between alternations, their effect is shown by the second stage of the curve.

UNIVERSity OF MichigaN.

The Path of a Rigid Electron Moving in a Magnetic Field of Constant Strength Rotating with Constant Angular Velocity.

By E. O. Hulburt.

A RIGID electron is assumed of mass $m$ and charge $e$. The reactions on its motion due to finite size, radiation, and the variation of mass with velocity are neglected. The electron is subjected to a magnetic field $H$ which rotates in the $X Z$ plane. The $X, Y$ and $Z$ components of the magnetic field are, respectively, $H \cos \omega t, o$, and $H \sin \omega t$, where $\omega / 2 \pi$ is the frequency of rotation. Let $x, y$ and $z$ be the positional coördinates of the electron. When Newtonian notation and electromagnetic units are used, the equations of motion of the electron are 